\title{
Increased knowledge transfer through the integration of research projects into university teaching
}

Oliver Vauderwange, Nicolas Javahiraly, Dan Curticapean

Oliver Vauderwange, Nicolas Javahiraly, Dan Curticapean, "Increased knowledge transfer through the integration of research projects into university teaching," Proc. SPIE 11143, Fifteenth Conference on Education and Training in Optics and Photonics: ETOP 2019, 111433T (2 July 2019); doi:

$10.1117 / 12.2523865$

SPIE Event: Fifteenth Conference on Education and Training in Optics and Photonics: ETOP 2019, 2019, Quebec City, Quebec, Canada 


\title{
Increased knowledge transfer through the integration of research projects into university teaching
}

\author{
Oliver Vauderwange* ${ }^{* a b}$, Nicolas Javahiraly ${ }^{\mathrm{b}}$ Dan Curticapean ${ }^{\mathrm{a}}$ \\ ${ }^{a}$ Offenburg University, Badstraße. 24, 77652 Offenburg, Germany \\ ${ }^{\mathrm{b}}$ University of Strasbourg / ICube, Boulevard Sébastien Brant, BP 10413, F-67412, Illkirch, France
}

\begin{abstract}
This paper describes the integration of the research project "Characterization of Color Vision using Spectroscopy and Nanotechnology: Application to Media Photonics" into an engineering course in the field of media technology. The aim is to develop the existing learning concept towards a more research-oriented teaching. Involving students in research projects as part of the learning process provides a deeper insight into current research topics and the key elements of scientific work. This makes it easier for students to recognize the importance of the acquired theoretical knowledge for the practice, which enables them to derive new insights of their own.
\end{abstract}

Keywords: research-oriented education, color vision, display technologies, learning scenario, optics and photonics.

\section{INTRODUCTION}

The mission of Offenburg University of Applied Sciences as an efficient educational institution is to enable its students to independently apply and further develop scientific knowledge and methods through qualified teaching. In order to fulfil these requirements, students of the Faculty of Media and Information are offered a wide range of courses with a broad spectrum of lectures in the fields of media informatics, media technology, media design and media economics. The teaching aim is the best possible qualification of the students on the basis of a strong scientific and research-oriented education, which also includes the development of important interdisciplinary competences.

In order to achieve this goal, we have developed a concept for research-oriented teaching that follows a defined strategy. For the success of this teaching approach it is crucial to offer a suitable learning environment. It is very important to use modern teaching methods and digital media to create an open and creative learning environment. In order to convey all necessary topics and competences clearly and intensively, we offer a mixture of theoretical lectures and practical work in the laboratories and in projects, combined with the advantages of modern digital media. This approach is now enhanced by a comprehensive integration of research projects into teaching.

\section{CONCEPT FOR RESEARCH-ORIENTED TEACHING}

The term "research-oriented teaching" describes a wide variety of courses that combine the fields of research and teaching. The central idea here is to familiarize students with current research topics and processes in their discipline in early semesters. The concept designed by the Faculty of Media and Information supports students in progressing from a comprehensible understanding of research topics, research processes, research methods and research results to autonomous research as far as possible. In this way, research-oriented teaching combines the theoretical and practical sides of the scientific knowledge process. In addition, the acquisition of specialist knowledge is combined with the acquisition and development of interdisciplinary competences. [1] [2] [3]

At the center of the concept is the general demand that research should be made visible and tangible for our students. Students are offered a concrete research context in courses in order to gain an insight into scientific work. On the basis of the "Research-Teaching Nexus", different teaching formats are offered to all teachers between lecturer and student activity as well as between the focal points research results and research processes/problems in order to implement different aspects of research-oriented teaching. Accompanied by various basic lectures, authentic, current, real or realistic problem situations are offered for independent study, which they work on with scientific methods and generate results. This enables them to immerse themselves in the world of subject-specific research. They are supported by the teacher, who supervises, advises and encourages them. The common communication between teachers and learners as

* oliver.vauderwange@ @s-offenburg.de; phone +49 781 205-4608; fax +49 781 205-110; www.hs-offenburg.de 
well as among students is an important element. Our concept and the underlying ideas are described in detail in the paper "Concept and development of research-oriented education in the university context". [1] [2] [3]

\section{PLANNED IMPLEMENTATION OF THE CONCEPT}

This paper describes the integration of the research project "Characterization of Color Vision using Spectrometry and Nanotechnology: Application to Media Photonics" into an established engineering course in the field of media technology. The current learning concept is to be further developed in the direction of research-oriented teaching in order to activate previously unused potentials. [4]

As already mentioned, a suitable learning environment is very important for the success of a defined learning scenario. This currently involves theoretical lectures, practical activities as well as practical applications and experiments embedded in a blended learning concept. The integration of current research projects is now the consequent continuation of the development process that this engineering course has undergone in recent years. The integration of students into research projects and research activities within the learning process offers a deeper insight into current research topics and key elements of scientific work. This makes it easier for students to recognize the importance of theoretical knowledge acquired in practice and to derive their own new insights from it. In the scientific knowledge process a combination of theory and practice takes place, which strongly supports the development of specialized knowledge and interdisciplinary competences. [5]

The primary aim of our educational program is to provide students with the best possible qualifications based on strong scientific and research-oriented education. By implementing our concept for research-oriented teaching, we intend to ensure that our students develop a deeper understanding of science and a positive research attitude. This includes the acquisition of methodical research competences and the ability to critically reflect on research activities and results. It is also important to gather experience in scientific collaborations with teaching researchers in order to be systematically and optimally prepared for future activities in research and development. [1] [2] [3] [5]

\subsection{The existing blended learning scenario}

The conception and development of the blended learning scenario for the engineering course "Media Technology: Color Representation and Display Devices", which is an integral part of the second and third semesters of the studies, was presented in a paper in 2016. [5]

Blended learning describes a teaching and learning scenario that provides a didactically meaningful correlation between attendance and online periods. Knowledge transfer and individual learning should be made easier. The students are oriented towards self-paced learning. There is a fixed timetable for regular lectures, the planned practical workshops and self-paced online learning. A central element of the scenario is the e-learning software moodle, which is used at the University of Offenburg. E-learning is an excellent way to add digital media to established teaching scenarios. Moodle is an internet-based open source software package for the development and implementation of e-learning courses under the GNU Public License. This online platform offers easy access to online learning materials with many interaction possibilities. Literature, scientific papers and documents are made available. Animations, videos and podcasts for the visualization of content are integrated as well as preparatory exercises, tasks and tests to check the level of knowledge. [5]

The engineering course "Media Technology: Color Representation and Digital Devices" is offered in semesters 2 and 3 in four consecutive segments. Particularly in the field of imaging and display technologies, we experience rapid technological progress that brings major challenges. In order to meet this progress, students need a broad theoretical knowledge. The specific theoretical basics are given in a defined lecture format. To deepen their knowledge, practical workshops are offered in the Media Technology Laboratory. A fixed schedule defines the attendance and online periods. Segment 1 is entitled "From light to color". The focus is on the scientific theories and knowledge of Newton, Maxwell, Hertz, Young, Brewster, Helmholtz and Hering. Segment 2 "Development of the color representation" focuses on colorimetry. It explains how human color perception is physically quantified and described. Segment 3 "From CIE Lab color space to color management" is clearly more application-oriented. The topic is ICC color management with all aspects such as ICC profiles, gamut mapping and rendering intents. And finally, segment 4, "Color representation on display devices", deals intensively with various display technologies and their applications. The discussion focuses on LCD technology and OLED technology. [5] [6] [7] [8] 
Three regular lectures per segment are used to impart theoretical knowledge. Participation is optional. These can be used by the students for face-to-face communication with the teacher. The online platform provides the complete contents of the lectures and exercises, which also enables students to work independently. In this online learning phase, the teacher only plays a minor role. Support is available online for students. However, personal on-site support is also possible through tutoring or small group sessions. In order to successfully complete a segment, the respective integrated practical workshop must be completed in the media laboratory. This is a must-attend event for all students. To be admitted, students must pass an online test to examine their level of knowledge. The results of the workshop must be published in a laboratory report and then presented to all participants with a poster. [5]

\subsection{Extension in the context of research-oriented teaching}

To be able to implement the planned concept for research-oriented teaching, the underlying structure of the engineering course will be adapted and supplemented with further teaching forms. An extended offer of additional seminars gives the students the opportunity to systematically familiarize themselves with the entire research process. A deepening of the content focus as well as the individual steps of the research process is enabled. Students should not only understand these, but should also be able to construct, apply and reflect on them. [5]

\section{Fixed Schedule over 2 Semesters}

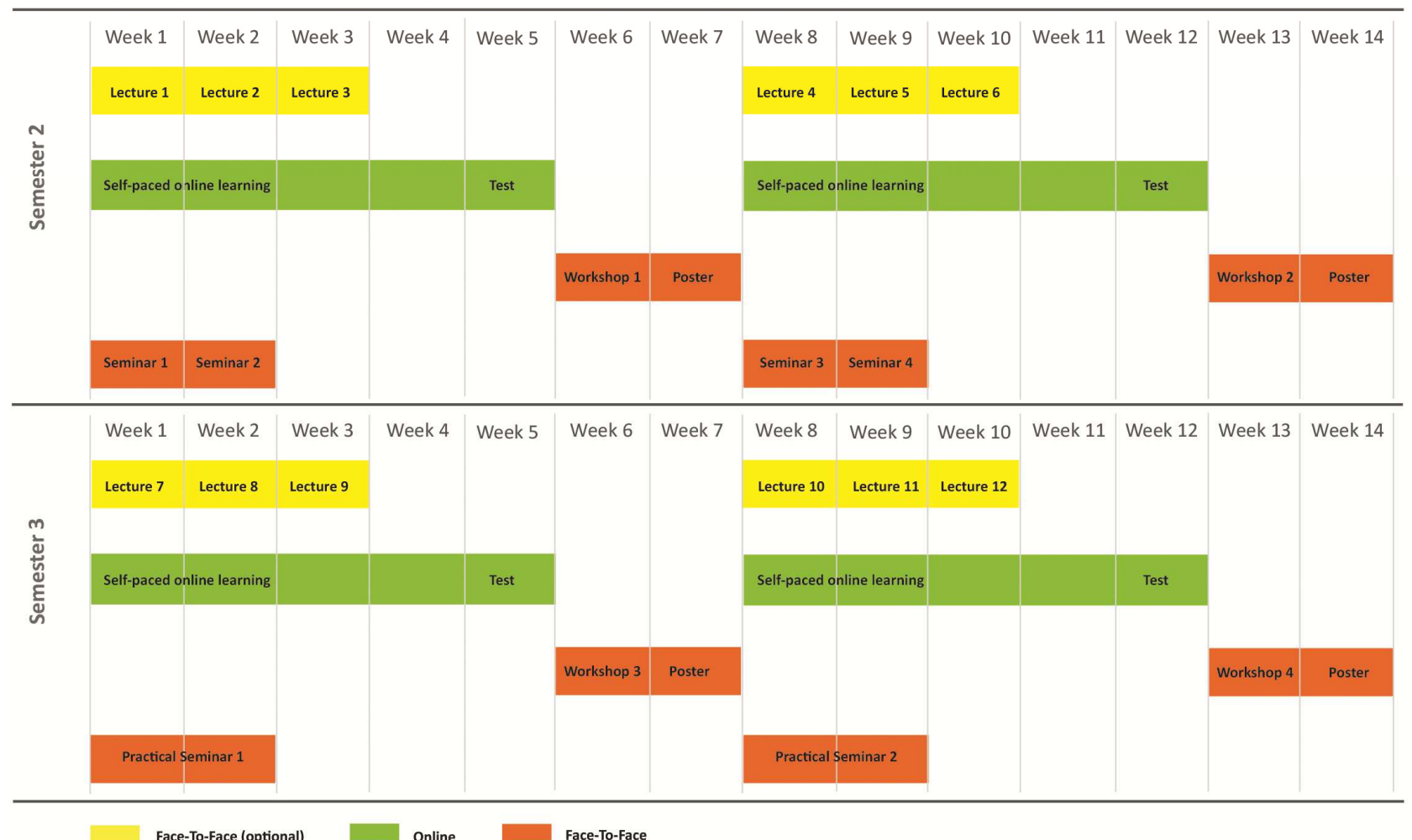

Figure 1. The extended schedule of the blended learning course „Media Technology: Color Representation and Display Devices". [5]

The program for semester 2, segment 1 "From light to color" and segment 2 "Development of color representation" will be expanded by four additional seminars (two per segment). These have a more theoretical focus and are designed to develop and encourage interdisciplinary skills. Seminar 1 is entitled "Scientific Writing". It supports the preparation of scientific papers within the framework of the studies. In addition to teaching the basics of scientific writing, the focus is on the use of the taught contents on the basis of selected and personal examples. In addition, the research and management of literature on scientific work is introduced to the program Citavi in its features and its handling. In seminar 2 "Presentation Technique and Rhetoric" the specific preparation for upcoming presentations or lectures is central. It is imparted, how projects and ideas are presented professionally and convincingly. Aspects such as oral pronunciation are considered just as safe and natural charisma of the presenter. In this seminar all important principles of 
the presentation preparation, connected with a variety of practical references and tools are imparted. Seminar 3 "Intuition and Creativity" has the goal to recognize and support creativity and intuition potentials. Students should understand, experience and explore creativity and intuition. To this end, creative environments will be designed in order to evaluate and use creative results. At the end of the accompanying seminar series in semester 2, the 4th seminar "Rational Decision Making" will be offered. With the emphasis on behavior and decision theory, the students are to be put into the position to question the own decision behavior. Basic techniques are presented to model decision situations and to resolve them in such a way that the decision can be formally justified. [5]

In the third semester, the seminars offered will be more practice- and research-oriented. These seminars take place in the Media Technology Laboratory on the basis of concrete tasks that are part of a current research project within the scope of a PhD thesis. Although the entire research cycle is not covered here, students can still experience different aspects of research. There will be the opportunity to apply methods in practice and to do research. The necessary freedom is given to make own decisions and thereby a critical analysis of the tasks to be performed is stimulated. In segment 3 , the metrological calibration and the characterization of the color rendering of displays are critically examined within the scope of the offered seminar. In segment 4, various color matching experiments will be performed, whose results will contribute to a better understanding of individual observers and their color perception. [4] [5]

\section{THE RESEARCH PROJECT}

The PhD project "Characterization of Color Vision by Spectrometry and Nanotechnology: Application for Media Photonics" is an international collaboration between the iCube-Institute (MACEPV groups) of the University of Strasbourg and the Faculty of Media and Information of the University of Offenburg. Specific characteristics of display technologies with respect to typical characteristics of human visual perception have been studied. [4]

\subsection{Research approach and formulated objectives}

Display and visualization devices are the interfaces between digital information and human perception, which transmit this information through light. Ideally, these interfaces should be optimized to meet the capabilities of human vision and visual cognition. This represents a major challenge, as a large number of different components are involved. These include, for example, the various display technologies, the used displays and their individually displayable color spaces, the colorimetric characterization and calibration of digital displays, the used measuring instruments, color management systems and software applications. An overall view of technology, measurement technology and workflow is therefore necessary in order to gain significant research results. [4]

The display technology in focus of this research are Quantum Dot (QD)-enhanced Liquid Crystal Displays (LCDs). TFT LCDs are still the dominant flat panel display technology today, revolutionized and revived by the integration of Quantum Dots into the backlight. This unique application of quantum physics provides an amazing performance boost. The range of displayable color space is significantly expanded and color saturation is increased, while display brightness and energy efficiency are improved. [4]

The research presented here discusses two main topics, Color Vision and QD-enhanced LCDs, and relates them directly to each other. It is based on the following three overarching questions:

1. How do QDs change the possibilities of color rendering in LCDs with a focus on the visual perception of the spectrum generated by these nanoparticles?

2. How do QDs expand the applications of displays with a focus on media photonics and scientific research?

3. Which perspectives does QD technology offer for the future?

To answer these questions, various focal points are set as the framework for the research work. Experimental tasks with display systems and the characterization of these original systems are performed. Colorimetric experiments help to develop a deeper understanding of the color vision of individual observers. Finally, the backlight technology of QDLCDs is used to study already used and alternative novel materials using simulation modeling. [4]

We have derived the following goals for this research work from the defined overriding questions and the focal points selected for answering them:

- A comparative study on the influence of QD-enhanced displays on visual perception.

- A comparative study on current measuring instruments (spectroscopy). 
- A comparative study of different colorimetric characterization models for the used QD-enhanced displays.

- The investigation of technical and optical components of displays that are responsible for color rendering.

- Analysis of the QD technology and its perspectives in the field of media photonics.

- The evaluation of expected improvements for media-related applications and scientific research applications.

\subsection{Organization and structure}

The first research topic deals with the question of how Quantum Dots (QDs) change the color rendering capabilities of LCDs, focusing on the visual perception of the spectrum produced by these nanoparticles in interaction with the backlight. Furthermore, the new wide gamut display technologies and especially the QD-enhanced display technology make the question of observer metamerism more important than ever. It is therefore necessary to deal with this topic in a systematic manner and to develop a deeper understanding of the color vision of the individual observer. [4]

A research design with colorimetric experiments was developed and a suitable research environment was created in the media technology laboratory at Offenburg University. The technical setup includes various test displays, various display calibration devices, a spectrometer for color and luminance measurement, and a computer with a defined software package for the digital control of the various devices. [4]

The colorimetric experiments are performed in three different steps:

1. Performance benchmarking of the test displays

2. Color characterization of test displays

3. Color matching experiments with individual observers

First, a performance benchmarking is carried out for all used displays. This requires a spectrometer, software for generating the test patterns and a power source analyzer. The aim is to check the performance of all selected displays. For this purpose, luminance, color space and power consumption of the displays are measured. The luminance efficiency and color space of the devices are compared. [4]

The second step is the colorimetric characterization of the used displays. This is an essential part of experimental methods of image processing and therefore of huge importance. First, the test displays are calibrated using a display calibration device. This step is necessary to achieve a defined state. The aim of this definition is to guarantee reproducible color reproduction for each further use of the display. On this basis, the actual color characterization of the screen can now be performed with a spectrometer. The performance of a characterization method for a particular screen is evaluated by predicting CIE XYZ values for a set of test patches (defined by RGB values). This prediction is then compared with the CIE XYZ values actually measured. A gray ramp and a color ramp are used as test patterns. As a result, we obtain conclusions about the characterization performance, which allow an evaluation of the used characterization model and a possible further development. [4]

For the concluding color matching experiments test patches based on the colorimetric fields of the X-Rite ColorChecker chart are used. The task for the observer is to match a color shown on the display in a defined size in an inserted mask as accurately as possible. Ideally, no color difference should be visible and measurable after color matching. The digital values are recorded for all colors and the spectral color stimuli are measured with a spectrometer. The evaluation of color differences is performed as standard in a reference system with a fixed observer (CIE 1931, CIE 1964). The data obtained provide an insight into the relationship between the visual evaluation and the measurement evaluation. All results of the color matching are now displayed in a diagram of the CIE a*b* level. In this way a comparison with the reference values can be carried out. This allows an exact evaluation of the expected intrapersonal and interpersonal variations. By assigning the achieved results to the displays used, a qualitative statement on the influence of the display technology used on the color perception of the individual observer is possible. [4]

The second research topic is the examination of the optical and technical components of displays, which have a decisive influence on the quality of color rendering. These material and technology-related studies are performed after the colorimetric experiments at the University of Strasbourg. The concept, organization and structure of the necessary studies are currently being developed. Different technical and optical components are responsible for the color rendering capability of a display. It is affected by various factors such as the spectral properties of the backlight, the transmission properties of color filters or the performance of the TFT panel. A concentration of research activities on the backlight technology is planned. Technical and optical components, used materials and nanoparticles are examined, dependencies, advantages, improvements are analyzed. Based on the results of the colorimetric experiments with different displays, 
three different backlight designs will be selected for further research. It is also very important to understand the physics behind the selected designs and materials. For this the optical properties of quantum dots will be analyzed by simulation modelling so that they can be compared with the results of the color matching experiments. [4]

\section{INTEGRATION OF THE RESEARCH PROJECT INTO SEMINARS}

Various aspects of the research project will be integrated into the seminars offered in the third semester. Although the entire research cycle will not be covered here, students will be able to experience different facets of research. These seminars offer the opportunity to report on a personal level to the students about own work as scientists. The research project as a whole can be considered and reflected.

Both seminars are conducted in the media technology laboratory. The research environment specially designed for the research project is used. The technical setup includes several available test displays, a spectrometer, four different display calibration devices, as well as a computer for the control of the devices and the required software applications. In addition, a power source analyzer and a multimedia controller are used. [4]

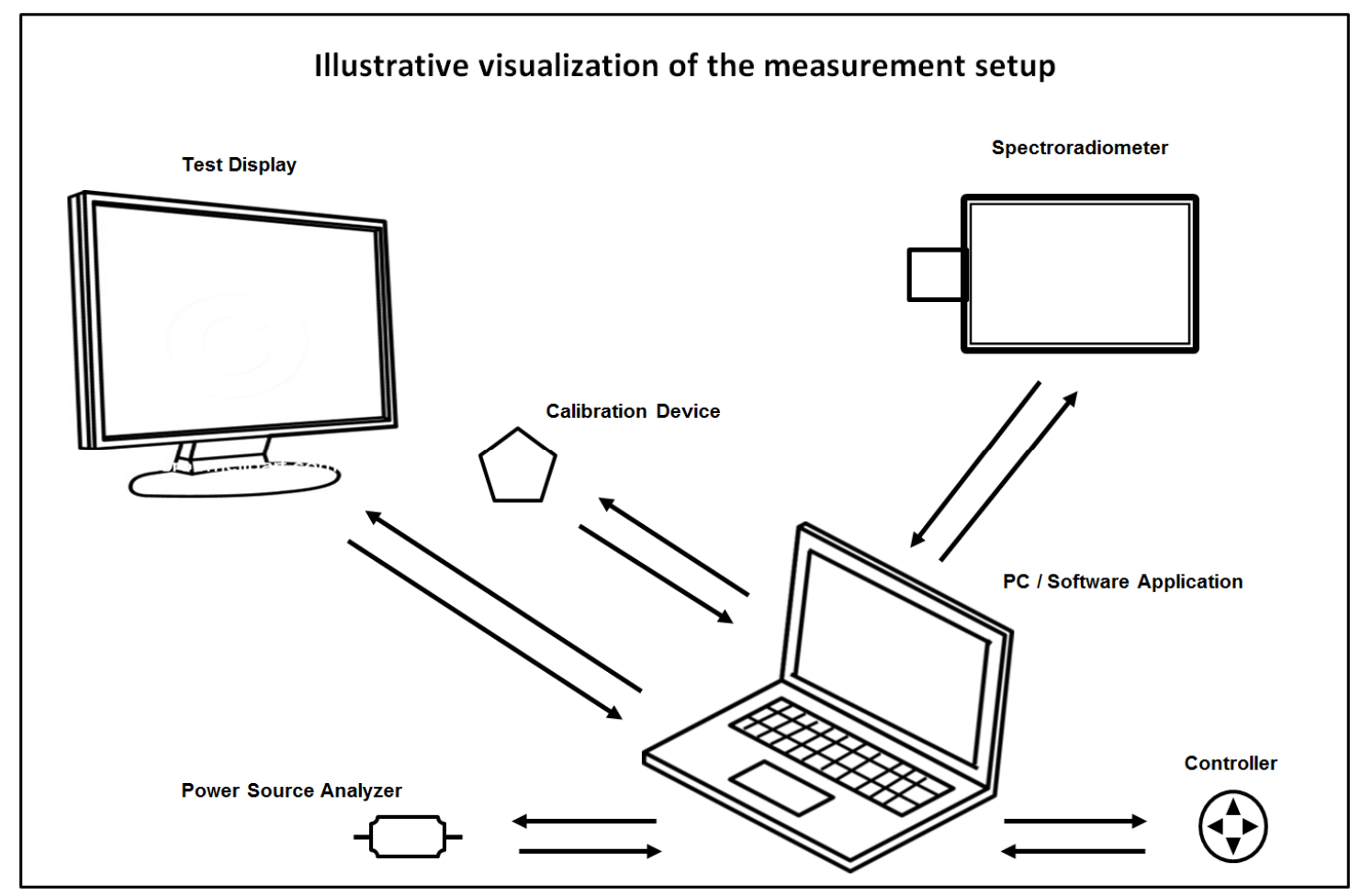

Figure 2. Illustrative visualization of the measurement setup. [4]

The seminar "ICC Color Management" will be integrated into segment 3. As the title indicates, there will be an intensive discussion on the topic of color management. The purpose of using a color management system is to achieve the best possible color consistency between the various input and output devices of a production workflow. Color rendering should be predictable and reproducible at all times. The importance of a standardized workflow in media production is demonstrated on the basis of various tasks. Two aspects of the colorimetric experiments of the research project will be integrated into the seminar. The basic requirement for a standardized color management workflow is the performed color calibration of the used displays. Prior to this, the students first perform a performance benchmarking of the used test displays. The acquired data can be used to evaluate the results of the research. They also enable us to conduct a longterm study on the performance of the displays. This is followed by the color calibration of the test displays. For this purpose, four different screen calibration devices are available. As part of the research project, a study is already being conducted to examine the influence of the used displays and color calibration devices on the quality of the color rendering and to objectively evaluate this. The acquired data can also be used for the evaluation of the study. [4] 
In the seminar "Color Matching" in segment 4, the identical color matching experiments planned within the framework of the research project will be performed. As already described in section 4.2, the task for the observer is to match a color shown on the display in a defined size in an inserted mask as accurately as possible. The stored digital color values and the measured spectral color stimuli are compared in a standardized reference system and possible color differences are determined. From the exact evaluation of the expected intrapersonal and interpersonal variations, conclusions can be drawn that contribute to a better understanding of individual observers and their color perception. In addition, by assigning the results achieved to the used displays, a qualitative statement on the influence of the used display technology on the color perception of the observer is possible. By continuing the experiments outside the research project, the amount of data obtained will be significantly increased, thus enabling continuous evaluation and updating of the results achieved in the research process. [4]

\section{PERSPECTIVE}

By expanding our teaching concept towards research-oriented teaching, we link the fields of research and teaching. Through the integration of the doctoral project "Characterization of color vision by spectrometry and nanotechnology": "Application for Media Photonics", students will be familiarized in the early semesters with the topic of research and scientific work. In our opinion, this implies that we integrate typical research activities as essential components of the learning process. We have therefore placed the general demand at the center of our concept that research should be made visible and tangible for our students. The students are offered a concrete research context in the courses in order to gain an insight into the scientific research. Through the extension towards research-oriented teaching, we hope to achieve an even higher motivation to deepen knowledge and to work independently within the framework of the offered teaching. This is the best precondition to achieve the best possible qualification of our students on the basis of a strong scientific and research-oriented education.

\section{REFERENCES}

[1] Huber, L., „Forschungsbasiertes, Forschungsorientiertes, Forschendes Lernen: Alles dasselbe?,“ Das Hochschulwesen, 62(1/2), pp 32-39 (2014).

[2] Healey, M., Jenkins, A., [Developing undergraduate research and inquiry], The Higher Education Academy, Heslington (2009).

[3] Sonntag, M., Rueß, J., Ebert, C., Friederici, K., Deike, W., „Forschendes Lernen im Seminar - Ein Leitfaden für Lehrende, " Humbold-Uni zu Berlin, 2016, <https://www.researchgate.net/publication/308047837_Forschendes_Lernen_im_Seminar_Ein_Leitfaden_fur _Lehrende> (27 April 2019).

[4] Vauderwange, O., Wozniak, P., Javahiraly, N., Curticapean, D., "New Perspectives in Color Vision using Spectroscopy and Nanotechnology: Application to Media Photonics," Frontiers in Optics 2017, OSA Technical Digest (online), (2017).

[5] Vauderwange, O., Wozniak, P., Javahiraly, N., Curticapean, D., "A blended learning concept for an engineering course in the field of color representation and display technologies," Proc. SPIE 9946, Optics Education and Outreach IV, 99460Y (2016).

[6] Vauderwange, O., Dreßler, P., Wozniak, P., Israel, K., Curticapean, D., "Active learning in optics and photonics - Liquid Crystal Display in the do-it-yourself," Proc. SPIE 9793, Education and Training in Optics and Photonics: ETOP 2015, 97930 Y (2015).

[7] Vauderwange, O., Dreßler, P., Wozniak, P., Curticapean, D., "Digital Devices: The big challenge in color management," Proc. SPIE 9188, Optics and Education Outreach III, 91880B (2014).

[8] Vauderwange O., Wielage H.-H., Haiss, U., Dreßler, P., Curticapean, D., "New frontiers in color management by using modern spectrometers," Proc. SPIE 9289, 12th Education and Training in Optics and Photonics Conference, 92891J (2013). 